\title{
Correlation between Multifractal Spectrum Based on Wavelet Leaders and T-Wave Alternans
}

\author{
R Cardo, A Corvalán \\ Universidad Nacional de General Sarmiento, Buenos Aires, Argentina
}

\begin{abstract}
In this work we analyze the correlation of the appearing T-wave alternans (TWA) with the shape (range) of the multifractal spectra of the ECG signals obtained with wavelet leaders based methods. A non-null correlation between the TWA occurring and lesser values for the Hölder exponent of maximum dimension has been observed, in agreement with the idea that the projection of TWA must have a non-despicable contribution in the higher scales of the multiresolution analysis of the signal. We are developing new algorithms capable to deal with non-concave multifractal spectra based in wavelet leaders projections, instead of existing methods that only gives concave spectra. These new algorithms will give a finer resolution of Hölder component of the signal and we hope that this richer information about the sharpness of the signal will allow better estimations of the magnitude of TWA and a closer relation between multifractal spectra and TWA occurring.
\end{abstract}

\section{Introduction}

The empirical evidence suggests that microvolt TWA tests are good predictors of the risk of sudden cardiac death (SCD). An interesting subject of study is the relation between these patterns and the analysis of the regularity of the signals of ECG performed with wavelet leaders. Sudden cardiac death (SCD) is one of the most important public health problems in the world today and is the leading cause of mortality in developing countries. Serious ventricular arrhythmias are the most common mechanism responsible for SCD. Thus, in order to prevent SCD, it is crucial to have effective diagnostic tools to identify patients at risk for these arrhythmias. Unfortunately, despite multiple trials in acute myocardial infarction (AMI) and congestive heart failure, our understanding of how to identify those patients at highest risk for SCD that seem to be related to the presence of the T-waves alternans and how to best prevent this devastating occurrence remains incomplete.

The study of the presence of T-waves alternans in an ECG seems to be strongly related to the distribution, density and dimension of the sets of the points of different regularity Hölder in terms of the dimension of correlation, and the promissory perspectives to warn the presence of the T-wave alternans from the Hölder exponents accumulated.

\subsection{Hölder exponents and multifractal spectra}

The pointwise Hölder exponents provide a natural enough way of quantifying the regularity of a function by means of a positive parameter that measures the major or minor ruggedness of the graph for every point. A function $f$ belongs to $C^{\alpha}\left(x_{0}\right)$ for $\alpha \geq 0$, if $\exists \mathrm{P}$ a polynomial of degree less than or equal to $[\alpha]$ ( the integer part of $\alpha$ ), and constants: $\quad C>0$ and $\delta>0$ such that if $x \in\left(x_{0}-\delta, x_{0}+\delta\right)$ then $\left|f(x)-P\left(x-x_{0}\right)\right| \leq C .\left|x-x_{0}\right|^{\alpha}$. So when $\alpha<1$ we obtain $\left|f(x)-f\left(x_{0}\right)\right| \leq C .\left|x-x_{0}\right|^{\alpha}$ for $x$ in some neighbourhood of $x_{0}$. Of this it is immediate that if $f \in C^{\alpha}\left(x_{0}\right)$ then $f \in C^{\alpha-\varepsilon}\left(x_{0}\right)$, for all $\varepsilon \geq 0$. Now we define the Hölder exponent of $f$ in $x_{0}$ as $H_{f}\left(x_{0}\right)=\sup \left\{\alpha: f \in C^{\alpha}\left(x_{0}\right)\right\}$. So we have that $f \in C^{\alpha}\left(x_{0}\right)$ for all $\alpha<H_{f}\left(x_{0}\right)$ and $f \notin C^{\alpha}\left(x_{0}\right)$ for $\alpha>H_{f}\left(x_{0}\right)$. For a continuous function in all points, we have that $H_{f}\left(x_{0}\right)$ define a function with values in $\Re \cup\{+\infty\}$ which say to us how the regularity of a function changes point to point. The Hölder exponents can change point to point giving very complex structures for the given signal. Then, the natural description of these structures is the distribution of the exponents in the range of regularity. More precisely, we can consider the dimensions of the point sets that have the same Hölder exponent. Since these sets in general have Lebesgue measure zero, the fractal dimensions are natural tools. The Multifractal Spectrum is $d(\alpha)=\operatorname{dim}_{H}\left\{x_{0}: H_{f}\left(x_{0}\right)\right\}$ defined as the Hausdorff dimension ( $\operatorname{dim}_{H}$ ) of points set $x_{0}$ having pointwise Hölder exponent $H_{f}\left(x_{0}\right)=\alpha$. 


\section{Methods}

\subsection{The wavelet leaders method [1]}

The procedure consists of seven steps:

1. For any couple $(j, k) \in N x Z$, let $I_{j, k}=\left[k 2^{-j},(k+1) 2^{-j}\right]$. Then, if $x \in R, \forall j \geq 1, \exists$ ! integer $k_{j, x}$ such that $x \in I_{j, k_{j, x}}=I_{j}(x)$ and let a wavelet $\psi \in C^{\infty}(R)$ a function in the Schwartz class, constructed in (Meyer, (1990)), then the set of functions $\left\{\psi_{j, k}=\psi\left(2^{j} \bullet-k\right\}_{(j, k) \in Z x Z}\right.$ forms an orthogonal wavelet basis (owb) of $L^{2}(R)$. Also, let $f \in C^{\varepsilon}(R)$ for some $\varepsilon>0$, and write it in the owb $f=\sum_{j, k \in Z}^{N_{s}} c_{j, k} \psi_{j, k}$ where $c_{j, k}$ is the wavelet coefficient of $f$ defined by:

$c_{j, k}:=c_{j, k}(f)=2^{j} \int f(t) \psi_{j, k}(t) d t$.

2. For any couple $(j, k) \in N x Z$, let us introduce the Wavelet Leader $L_{j, k}$ associated with $f$ and $\psi: L_{j, k}=\sup _{j^{\prime} \geq^{\prime} j, k^{\prime} 2^{-j} \in I_{j, k}}\left|c_{j^{\prime}, k^{\prime}}\right|$.

3. Then, with any point $x \in R$ and any scale $j \geq 0$ can be associated the coefficient: $L_{j}\left(x_{0}\right)=\sup L_{j, k}$.

$$
\left|k-k_{j, x}\right| \leq 1
$$

4. A theorem about the pointwise Hölder exponent proved in Jaffard (2004) affirms that: $h_{f}\left(x_{0}\right)=\liminf _{j \rightarrow+\infty} \frac{\log \left(L_{j}\left(x_{0}\right)\right)}{\log \left(2^{-j}\right)}$.

5. Recall that the Legendre Transform of a function $\varphi: q \in R \rightarrow \varphi(q)$ is the mapping

$\varphi^{*}: h \in R \rightarrow \varphi^{*}(h) \in R \cup\{-\infty\} \quad$ defined by $\varphi^{*}(h)=\inf _{q \in R}(q h-\varphi(q))$.

6. For any function $f \in L^{2}(R)$ decomposed in the before equation, we can introduce the scaling function $\xi_{f}: p \in R \rightarrow \xi_{f}(p)$ associated with $f$ as follow: $\xi_{f}(p)=\liminf _{j \rightarrow+\infty} \frac{-1}{j} \log \sum_{k \in Z}^{*}\left|L_{j, k}\right|^{p}$ where $*$ means that the sum is taken over the $k$ such that $\left|L_{j, k}\right|$ does not vanish. So, for each $j \geq 1$ the function $p \rightarrow \sum_{k \in Z}^{*}\left|L_{j, k}\right|^{p}$ is log-convex and non-increasing when $j$ is large enough, then the mapping $\xi_{f}$ is concave and non-decreasing on $R$ (as limit of the infimum of non-decreasing concave functions).

7. This kind of free energy functions are naturally introduced to formulate a multifractal formalism for functions based on the representation as wavelet series (see Jaffard, (1997)). Frisch and Parisi first proposed a formula (that links multifractal spectrum of a function $f$ with some averaged quantities derived from $f$ ): generally called the Frisch-Parisi's conjecture, that can be generalized and reformulated (see Frisch and Parisi, (1985)), if we call $d_{f}(h)$ to the Hausdorff dimension of set $E_{h}{ }^{f}:=\left\{x: h_{f}(x)=h\right\}$, then:

$$
d_{f}(h)=\inf _{p>0}\left(p h-\eta_{f}(p)\right)=\eta_{f}{ }^{*}(h) \text { where the }
$$

mapping $\eta_{f}: p \in R \rightarrow R \cup\{-\infty\}$ is a suitable free energy function associated with $f$. Then, (Jaffard, (2004)) established that: the scaling function $\xi_{f}$ depends only on $\mathrm{f}$, NOT on $\psi$, and if $f$ satisfies the multifractal formalism at the exponent $h>0, \eta_{f}=\xi_{f}$. Now we define the Hausdorff Multifractal Spectrum of $f$ by $d_{f}: h \rightarrow d_{f}(h)=\operatorname{dim}\left(E_{h}{ }^{f}\right)=\left(\xi_{f}\right)^{*}(h)$.

Hölder estimation by means of the wavelet leaders: The pointwise Hölder exponents of a function can be estimated studying the decay of the wavelet leaders in the cone of influence of every point.

Actually, it is well known the characterization of spaces of functions by means of the wavelet coefficients of $f$ :

$$
f \in C^{\alpha}\left(x_{0}\right):\left|c_{j, k}\right| \leq C \cdot 2^{-\alpha j} \cdot\left(1+\left|2^{j} \cdot x_{0}-k\right|\right)^{\alpha}
$$

for some constant $C$, and with a weaker reciprocal (there appears a factor of logarithmic decay involved). In this line, under general enough hypothesis it is possible to obtain the pointwise Hölder exponent if one assumes that essentially: $\left|c_{j, k}\right| \approx K .2^{-H_{f}\left(x_{0}\right) j}$ and taking $\log _{2}$, we obtain: $\lim _{j \rightarrow+\infty} \frac{\log _{2}\left|c_{j, k}\right|}{-j}=H_{f}\left(x_{0}\right)$. The above mentioned procedure - resides of specifies presumptions that its application needs on a discreet series - suffers from disadvantages when the signal shows oscillating 
singularities (v. g. chirps). When a time-frequency singularity lies on small intervals, great values of $K$ are necessary, and then they cannot be scorned even at the maximum scale that the discretization admits. This cannot be avoided since the constants involve (after fixing a scale) both $C$ and the factor $\left(1+\left|2^{j} \cdot x_{0}-k\right|\right)^{\alpha}$, and this introduces instabilities in these estimations. On the other hand, by considering only the wavelet leaders, the bounds are unified in all the intervals of the cone of influence - that alludes also to the contiguous intervals in every scale close to $x_{0}-$, and then the perturbations introduced by the oscillations decrease. This allows to formulate the approximation of the law of decay without the factor that involves the translations: $\left|L_{j, k}\right| \approx K .2^{-\alpha j}$. Then we do an extrapolation to the limit to estimate: $H_{f}\left(x_{0}\right)=\lim _{j \rightarrow+\infty} \frac{\log _{2}\left|L_{j, k}\right|}{-j}$. This provides a faithful estimation of the pointwise Hölder exponents that does not produce great variations, for example, in the presence of chirps of the signal.

\subsection{Gradient modulus wavelet leaders projection method}

Turiel, A. et al (2006) [2] use an histogram to estimate the density of the Hölder exponents from the values for the smallest scale, by considering the coefficients: $c_{j, k}:=c_{j, k}(f)=2^{j} \int f(t) \psi_{j, k}(t) d t$

for $\varepsilon_{0}=2^{-j_{0}} \approx 0$ (Gradient Modulus Wavelet Projection). Our proposal is to estimate with a direct method, similar to the above mentioned (Turiel), based on the behavior of the wavelet leaders $L_{j, k}$ instead of the $c_{j, k}$. In this way the stability in the face of to oscillating singularities provided by the wavelet leaders is preserved; moreover we also have the freedom of the Turiel method (without the Legendre transform) to about the concavity or non-concavity of the multifractal spectrum. Then we will use the abbreviation: GMWLP (Gradient Modulus Wavelet Leaders Projection), for the algorithm of direct estimation of the multifractal spectrum with wavelet leaders, which four steps we resume as follows:

1. We estimate the Hölder exponents extrapolating at limit: $j=N,\left(\frac{N}{2}\right),\left(\frac{N}{2^{2}}\right), \ldots,\left(\frac{N}{2^{\log _{2}(N)}}\right) \rightarrow 0, \quad$ and $\quad$ we obtain $h_{f}\left(x_{0}\right)=\lim _{j \rightarrow+\infty} \frac{\log _{2}\left|L_{j, k}\right|}{-j}$.

2. We estimate the density $\rho(\alpha)$ : Theoretically $\rho(\alpha)$ is a density function, that is the derivative of the distribution (probability) function $F$, such that $F(\alpha)=P(x \leq \alpha)=\int_{-\infty}^{\alpha} p(t) d t$, and then:

$p(\alpha)=\frac{d(F(\alpha))}{d \alpha}=\frac{d\left(\int_{-\infty}^{\alpha} p(t) d t\right)}{d \alpha}=\frac{d(P(x \leq \alpha))}{d \alpha}=\lim _{\varepsilon \rightarrow 0} \frac{P(x \leq \alpha+\varepsilon)-P(\alpha-\varepsilon)}{2 \varepsilon}$

So we take a partition as fine as possible of $\left[\alpha_{\text {min }}, \alpha_{\text {máx }}\right]$ and we count the proportion of the points of the series which Hölder exponent lies in each interval: $p(\alpha) \approx\left(\frac{1}{2 \varepsilon}\right)\left(\frac{\# \text { point } s \text { with } \alpha-\varepsilon \leq H_{f}(x) \leq \alpha+\varepsilon}{\# \text { total point } s \text { of the serie }}\right)$

3. We estimate the dimension of each $\alpha$ (of the partition): $\operatorname{dim}\left(E_{\alpha}\right)=\lim _{r \rightarrow 0} 1-\frac{\log \left(\frac{\rho(\alpha)}{\rho\left(\alpha_{1}\right)}\right)}{\log (r)}$, where $E_{\alpha_{1}}$ is the set of maximum density and $r$ is the ratio of the intervals of the mesh: Frequently the series has full support (dimension 1), and then the set $E_{\alpha_{1}}$ of major density must also has dimension 1 .

4. On the other hand, for little scales $(r \approx 0)$ we approximate: $\operatorname{dim}\left(E_{\alpha}\right) \approx 1-\frac{\log \left(\frac{\rho(\alpha)}{\rho\left(\alpha_{1}\right)}\right)}{\log (r)}=\operatorname{dim}\left(E_{\alpha_{1}}\right)-\log _{r}\left(\frac{\rho(\alpha)}{\rho\left(\alpha_{1}\right)}\right)$ and consequently, $\frac{\rho\left(\alpha_{1}\right)}{\rho(\alpha)} \approx \frac{r^{\operatorname{dim}\left(E_{\alpha}\right)}}{r^{\operatorname{dim}\left(E_{\alpha_{1}}\right)}}$, as one expect for any reasonable definition of dimension Though the GMWLP is preferable on the GMWP, both methods only differ in the way of estimating the pointwise Hölder exponents, and then they follow a common scheme:

Discretization of the interval: $\left[\alpha_{\text {min }}, \alpha_{\text {máx }}\right]: \alpha_{\text {min }}=\alpha_{0}, \alpha_{1}, \ldots \ldots, \alpha_{n-1}, \alpha_{n}=\alpha_{\text {máx }}$.

Approximate evaluation of the density function of the pointwise Hölder exponents in the nodes of the partition: $\rho\left(\alpha_{i}\right)$. Estimation of the dimensions of the sets of each pointwise Hölder exponent: $\operatorname{dim}\left(E_{\alpha_{i}}\right)$.

\section{Results}

The results of the five entries of the TWA-Challenge of PhysioNet confirms the fact that there are a strong correlation between the difference of the multifractal spectra and their concave hulls with a high level of significance, but it is not perhaps sufficient for being the 
basis of a method of systematic detection of TWA. Notwithstanding it is an interesting subject of study the causes of this correlation.

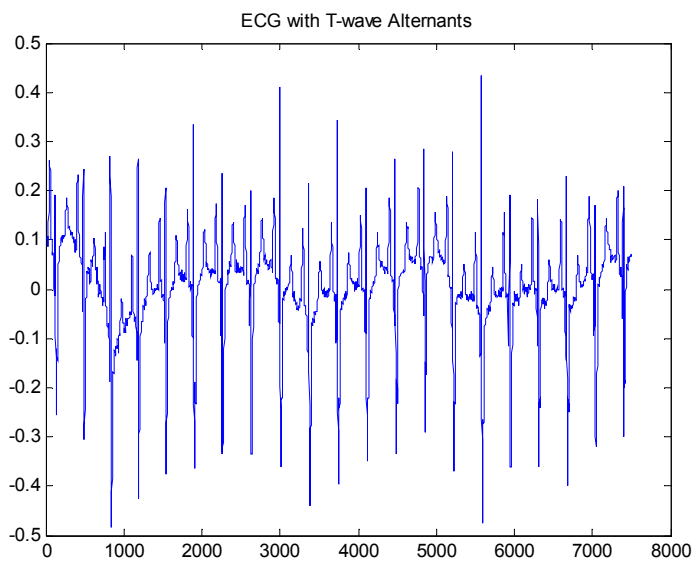

Figure 1. An example of Electrocardiogram series.

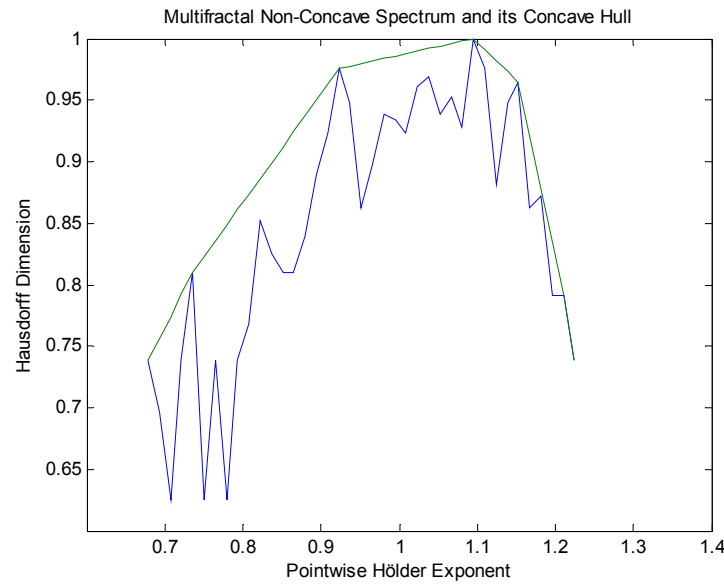

Figure 2. Multifractal spectrum and its concave hull referred to series of figure 1 .

\subsection{Computational complexity of wavelets based methods}

The most expensive step is the computation of wavelet coefficients requiring an order of about $\mathrm{N} \cdot \log (\mathrm{N}) \cdot K$ products where $\mathrm{K}$ is a proportional constant from the number of nodes necessaries for implementing the mother wavelet. With exception of when there are reasons to expect high regularity it is sufficient to take (for example, in our implementation) a wavelet of compact support of the fourth order: We use the nearsymmetric Daubechies wavelet: $\mathrm{Sym} 4$, with $\mathrm{K}=4.4$ - 1 $=15$. This step only is needed once. Other usual methods for computing the multifractal spectrum (not wavelet based) require typically about $\mathrm{O}\left(\mathrm{N}^{2}\right)$ operations.

\section{Discussion and conclusions}

The patterns of many physiological processes exhibit a strong correlation with the regularity of the signals of measurements related with them and consequently with their multifractal spectra. Some examples are the series of EEG before and during epileptic crisis of brain absences, series of human gait in healthy and ill persons, heartinterbeat signals in human races [3,4], etc. This suggested us the possibility of a relation between some characteristic of ECGs - normal and pathological- and their spectra, and specifically, the detection of T-wave alternans, because the relation of time-scale analysis and frequency spectrum (where TWA should be reflected) and Hölder regularity -above mentioned-. An interesting question is the use of GMWLP method that can detect non-concave spectra. Such non-concavity may be relevant in processes with superposition of contributions of many sources, and we thought that it can have influence in this problem. To consider this last item we compute the norm in L1 of the difference between the multifractal spectra computed with the GMWLP, and their concave hull. Also we want to mention that the results of the entry indicate that the non-concavity of the spectra, alone, presents a higher correlation than the concave hull of them (computed with the WL), and also higher than a weighted average of the index of nonconcavity and the mean value of the Hölder exponents.

\section{Acknowledgements}

We are grateful to God for Gary and Bill Sanders and our other benefactors for their invaluable help.

\section{References}

[1] Jaffard, S., Wavelet techniques in multifractal analysis, (2004), fractal geometry and applications, Proceedings of Symposia in Pure Mathematics, AMS, Providence, RI

[2] Turiel, A., Pérez-Vicente, C., Grazzini,J., Numerical methods for the estimation of multifractal singularity spectra on sampled data: A comparative study (2006)

[3] Eva Wesfreid, Véronique L. Billat, Yves Meyer, Multifractal analysis of heartbeat time series in human races, Elsevier Science. 2004 Dec. 26

[4] Munoz-Diosdado, A. del Rio Correa, J.L. Brown, A.F., Multifractality in time series of human gait, Engineering in Medicine and Biology Society, 2003. Proceedings of the 25th Annual International Conference of the IEEE

Romina Cardo- Álvaro Corvalán

Instituto del Desarrollo Humano. Universidad Nacional de General Sarmiento, José María Gutiérrez 1150, Los Polvorines (B1613GSX), Pcia de Bs As, República Argentina rcardo@ungs.edu.ar acorvala@ungs.edu.ar 